\title{
SEPTO-OPTIC DYSPLASIA AND CORTICAL MALFORMATIONS
}

Three children with septo-optic dysplasia (SOD) and an associated malformation of cortical organization are reported from McGill University and the Montreal Children's Hospital, Quebec, Canada. Case 1, a 10-year-old girl, presenting with left hemiparesis, deafness and short stature, was microcephalic, developmentally delayed, and had a growth hormone deficiency. MRI showed an absent septum pellucidum, absent cochlea, hypoplasia of the optic chiasm, and right sylvian polymicrogyria. Case 2, a 4-year-old boy, had absent visual fixation and global developmental delay. MRI showed absent septum pellucidum, optic chiasm hypoplasia, and focal polymicrogyria. Case 3, a 4-year-old girl, had global developmental delay, spastic quadriparesis, and facial diplegia with dysarthria and drooling. MRI showed absent septum pellucidum, optic chiasm hypoplasia, and right open-lipped schizencephaly. The term SOD-Plus is proposed, to extend the SOD-schizencephaly syndrome to include other associated cortical malformations. (Miller SP, Shevell MI, Patenaude Y, Poulin C, O'Gorman AM. Septooptic dysplasia plus: a spectrum of malformations of cortical development. Neurology April (2 of 2) 2000;54:1701-1703). (Reprints: Dr M Shevell, The Montreal Children's Hospital, Room A514, 2300 Tupper Street, Montreal, Quebec, H3H 1P3, Canada).

COMMENT. Previous reports of septo-optic dysplasia (SOD) have emphasized the common association with schizencephaly, and the occurrence of alternative or additional cortical malformations may be under-recognized. SOD presenting with global developmental delay or spastic motor deficits should prompt screening with MRI to identify polymicrogyria and other cortical malformations. The most common clinical presentation of SOD includes visual impairment and signs of pituitary hypofunction. The MRI abnormalities may not be limited to schizencephaly.

\section{CONTINUUM OF BRAIN ABNORMALITIES IN FETAL ALCOHOL SYNDROME}

The structural and functional integrity of the brain was assessed by MRI and positron emission tomography (PET) in 19 young adults with fetal alcohol syndrome (FAS) examined at the University of British Columbia, Vancouver. The majority was nonretarded, and the Full Scale IQ ranged from 66 to 92 (mean, 80). MRI was definitely abnormal in only one patient, showing hypoplasia of the corpus callosum. In one other, the left occipital lobe was lower than the right, and also appeared hypometabolic on the PET scan. Decreases in reiative regional cerebral metabolic rates were significantly abnormal in 5 brain regions involving the thalami, caudate heads, and the right caudate/putamen body. The patient with corpus callosal hypoplasia had the lowest IQ (66). A continuum of neuropathology in FAS is suggested. (Clark CM, Li D, Conry J, Conry R, Loock C. Structural and functional brain integrity of fetal alcohol syndrome in nonretarded cases. Pediatrics May 2000;105:1096-1099). (Respond: Campbell M Clark PhD, Department of Psychiatry, University of British Columbia, 2255 Wesbrook Mall, Vancouver, BC V6T 2A1, Canada).

COMMENT. Previous reports of cerebellar atrophy and dysgenesis of the corpus callosum represent cases of severe FAS, whereas the current study findings of mainly regional changes in cerebral metabolic rates are characteristic of nonretarded cases. A continuum of neurotoxic effects of alcohol on the developing fetal brain is suggested. 